\title{
Review Article \\ The MicroRNAs as Prognostic Biomarkers for Survival in Esophageal Cancer: A Meta-Analysis
}

\author{
Wenbo Fu, ${ }^{1,2}$ Lijuan Pang, ${ }^{3}$ Yunzhao Chen, ${ }^{3}$ Lan Yang, ${ }^{3}$ Janbo Zhu, ${ }^{3}$ and Yutao Wei ${ }^{2,4}$ \\ ${ }^{1}$ Medical College of Shihezi University, Shihezi, Xinjiang 832000, China \\ ${ }^{2}$ Department of Thoracic and Cardiovascular Surgery, Hospital of Xinjiang Production and Construction Corps, \\ Urumchi, Xinjiang 830002, China \\ ${ }^{3}$ Department of Pathology, Medical College of Shihezi University, Shihezi, Xinjiang 832000, China \\ ${ }^{4}$ Department of Thoracic and Cardiovascular Surgery, First Hospital Affiliated to Medical College of Shihezi University, \\ Shihezi, Xinjiang 832000, China \\ Correspondence should be addressed to Yutao Wei; wytfwb@126.com
}

Received 14 March 2014; Revised 24 May 2014; Accepted 18 June 2014; Published 6 July 2014

Academic Editor: Manuela Ferracin

Copyright (c) 2014 Wenbo Fu et al. This is an open access article distributed under the Creative Commons Attribution License, which permits unrestricted use, distribution, and reproduction in any medium, provided the original work is properly cited.

\begin{abstract}
Objectives. We performed this meta-analysis to summarize all the results from available studies, aiming delineating the prognostic role of miRNA in esophageal cancer. Design and Methods. We searched the electronic databases PubMed, EMBASE, and ISI Web of Science without time restrictions for the correlative literature to aggregate the survival results. Relevant data were extracted from studies investigating the relationship between miRNAs expression and survival in esophageal cancer patients. Pooled hazard ratios of miR-21and miR-375 for OS in ESCC were calculated. Results. A total of 25 studies involving 2,258 subjects analyzed the relationship between miRNA and prognosis of EC. In all, thirty-nine miRNAs associated with prognosis were reported in these studies. The pooled HR of higher miR-21 expression compared with lower miR-21 expression in ESCC was 1.84 (95\% CI: 1.41-2.40, $P<0.001$ ), which could significantly predict poorer OS in ESCC. Besides, higher miR-375 was also a significant predictor for OS in ESCC, with a pooled HR of 0.55 (95\% CI: 0.42-0.72, $P<0.001$ ). Conclusions. Our results support that miR-21 and miR-375 have a prognostic role in ESCC and may be useful therapeutic targets for the treatment of ESCC and meticulous follow-up for early detection of recurrence.
\end{abstract}

\section{Introduction}

Esophageal cancer (EC) represents an interesting but a very lethal group of malignancies associated with varied risk factors and geographical distribution [1]. EC is the sixth most common cancer and the sixth leading cause of cancer death in human worldwide, affecting men more than women [2]. According to a new study, there were estimated 482,300 new EC cases and 406,800 EC-caused deaths in 2008 worldwide [3]. The deadly disease is often diagnosed at later stages, and the survival rate for affected patients is very low, ranging from $10 \%$ in Europe [4] to $20-30 \%$ in Asia [5], so that the prognosis of affected patients is unsatisfactory, despite the development of therapeutic options such as surgery, chemotherapy, and radiotherapy, highlighting the need to identify novel biomarkers for early detection and prognostic classification [6].

Recently, microRNAs (miRNAs) have attracted attention for their involvement in cell development, differentiation, proliferation, and apoptosis by targeting mRNAs for cleavage or translational repression at the posttranscriptional level [7]. The miRNAs are a class of small, approximately 23 nucleotide noncoding RNAs that are highly conserved and endogenously expressed across mammals and other species [8]. These little molecules are transcribed in the nucleus and are subsequently cleaved by the class II RNase III enzyme Drosha to produce pre-miRNAs [9]; these premiRNAs are then exported to the cytoplasm where the RNase III enzyme Dicer further processes them into mature miRNAs [7]. They act as part of a multiprotein complex to 
silence or repress the expression of target genes [10] and have emerged as a key component of posttranscriptional regulation [11]. Calin et al. [12] demonstrated that more than $50 \%$ of miRNA genes are located in fragile sites and cancerassociated genomic regions, suggesting that miRNAs may play a vital role in the pathogenesis of human cancers and are able to dramatically change the biological function of organisms. Growing evidence has indicated that aberrant expression of miRNAs has been linked to development and progression of cancer and has been shown to have prognostic significance in several tumor types, including colon [13], lung [14], breast [15], and ovarian cancer [16]. Recent studies showed that miRNAs are associated with prognosis in EC as well, suggesting that they could be developed as prognostic classifiers to guide therapeutic decisions [17, 18]. We performed the meta-analysis of the data available from articles published in this field with the main aim of evaluating the role of specific microRNA as a prognostic biomarker in EC.

\section{Material and Methods}

This meta-analysis followed the guidelines of the Metaanalysis of Observational Studies in Epidemiology group (MOOSE) issued by Stroup et al. [19] and Preferred Reporting Items for Systematic Reviews and Meta-analysis (PRISMA) criteria by Moher et al. [20].

2.1. Publication Search. We carefully searched the PubMed, EMBASE, and ISI Web of Science databases using the search terms "miRNA," "microRNA," "esophageal cancer," "esophageal neoplasm," "esophageal carcinoma," "ESCC," "prognos*," and "surviv*" updated until January 15, 2014, and limited to English language papers. Furthermore, reference lists of retrieved articles and review articles were reviewed manually to identify missing relevant publications.

2.2. Eligibility Criteria. Two reviewers (Pang and Chen) independently assessed eligibility of the retrieved articles. The studies were included in the meta-analysis if they reported survival data in esophageal cancer patients, provided sufficient data for determining an estimate of $\mathrm{HR}$ and a $95 \% \mathrm{CI}$, and enrolled more than 20 patients, and study patients did not overlap with patients in other included articles. We did not assign each study a quality score, because no such score has received general agreement for use in a prognostic metaanalysis, especially of observational studies [21].

2.3. Data Extraction. Two reviewers (Zhu and Wei) independently extracted the data of the included studies. The following data was extracted: name of first author, year of publication, country, number of participants, study design, tumor stage, HR with $95 \% \mathrm{CI}$, and so forth. In the study reported by Zhao and colleagues [17], no HR or 95\% CI were available; thus the HR and 95\% CI were estimated from the $P$ value of the log rank test and the number of observed events in each group, or the Kaplan-Meier curve [22].
2.4. Statistical Analyses. For miRNAs evaluated appropriately in a single study, the summary HR (including 95\% CI) represents the value reported in that study. For miRNAs that were evaluated appropriately in multiple studies, fixed effects summary HR and 95\% CI were calculated by using the generic inverse variance method and the random effects model according to the D-L method [23]. Interstudy heterogeneity was assessed by the $I^{2}$ statistic [24]. By convention, an observed HR $>1$ indicated worse outcome for the study group relative to the reference group and would be considered statistically significant if the $95 \%$ confidence interval did not overlap 1, with $P<0.05$ [25]. Articles that presented insufficient data to derive the HR and CI for OS and PFS/RFS were included in the systematic review (qualitative analysis) but excluded from the meta-analysis (quantitative analysis) [26]. Publication bias was weighted by Begg's funnel plot with the Egger's bias indicator test with $P<0.05$ being considered statistically significant [27]. All of the analyses were performed using STATA vll.0 (Stata Corp., College Station, TX) [28].

\section{Result}

Two-hundred and eighty-seven studies for miRNAs associated with prognosis were identified from a primary literature search in PubMed, EMBASE, and ISI Web of Science databases. After manually screening the titles, abstracts, and key data, two-hundred and sixty-six records were excluded because they were either duplicate, review articles, or irrelevant to the current study. Twenty-seven articles were further reviewed in detail. Two articles were further excluded because data were unavailable for further analysis $[29,30]$. A total of 25 studies involving 2,258 subjects analyzed the relationship between miRNA and prognosis of EC (data was not shown). In all, thirty-nine miRNAs associated with prognosis were reported in these studies and miR-21, miR-145, and miR375 were reported by more than three articles. There are four studies investigating the relationship between miR-145 and prognosis of EC. Pooled HR and 95\% CI for miR$21 / 375$ to predict OS in ESCC were calculated. Data were not synthesized for miR-145 because of different EC histology and types of survival analysis. Clinicopathological characteristics of eligible studies are summarized in Table 1. For miR-21, 5 of 7 studies were conducted in Asian population, 1 derived from multipopulation, and 1 study was performed in western population, which may lead to a discrepancy of ethnicity. One study included 50 patients, 3 studies examined between 51 and 100 individuals, and 3 studies examined between 100 and 200 individuals. Cancerous tissues were usually examined to determine miRNAs expression level, while plasma or serum samples were also tested in two studies [31,32]. For miR375, 4 of 6 studies were conducted in Asian population, 1 derived from multipopulation, and 1 study was performed in western population. One study included 50 patients, 2 studies examined between 51 and 100 individuals, and 3 studies examined between 100 and 200 individuals. Four studies investigate the relationship between miR-145 and prognosis of EC. All of the studies were retrospective in design. Notably, 


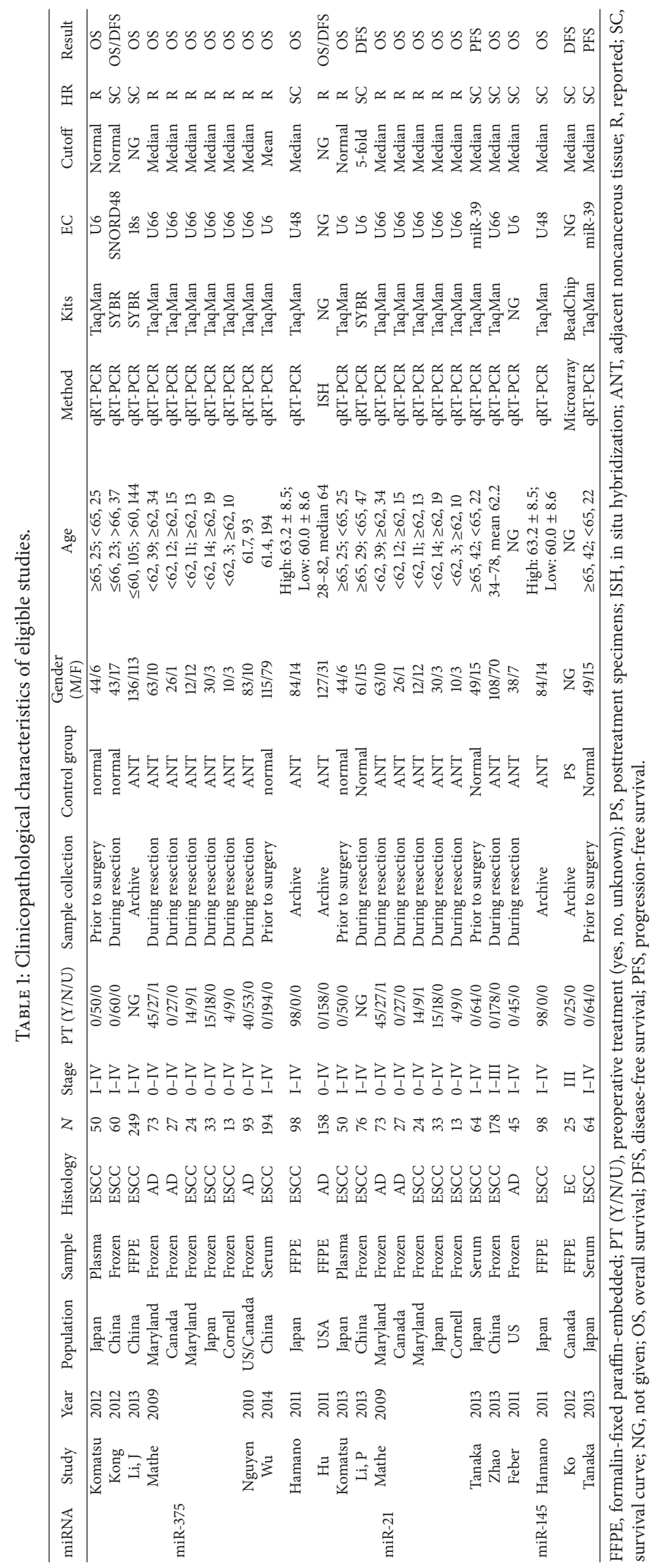


the cutoff values of miRNAs were different in each study, most with median or mean. More detailed information was summarized in Table 1.

For studies evaluating OS in ESCC, no significant heterogeneity have been found (for miR-21: $I^{2}=0.00 \%, P=0.26$; miR-375: $I^{2}=0.00 \%, P=0.13$ ), so that the fixed effects were applied to calculate the pooled HR (pooled $\mathrm{HR}=1.84,95 \%$ CI 1.41-2.40 $P<0.001$ for miR-21, Figure 1; and 0.55, 95\% CI: $0.42-0.72, P<0.001$ for miR-375, Figure 2). Publication bias of the included studies was evaluated by Begg's plots and Egger's test. No significant publication biases were found in the results of Begg's plots of miR-21 and miR-375. Egger test reached similar conclusions. Pooled HR and 95\% CI were not calculated for miR-145 because of different EC histology and types of survival analysis.

\section{Discussion}

In response to the need for independently prognostic molecular markers for ESCC that are readily assayable on routinely acquired clinical specimens, we conducted this analysis of the published esophageal cancer literature to identify a group of miRNAs for which the data support validation as prognostic biomarkers of esophageal cancer outcomes. In this first extensive systematic review about miRNAs in esophageal cancer, 25 studies involving 2,258 subjects analyzed the relationship between miRNA and prognosis of esophageal cancer (data was not shown). A total of 39 miRNAs involved in the prognosis of esophageal cancer were reported in these studies. Most miRNAs were identified by one single study and only 3 miRNAs (miR-21,375,145) were reported by more than 3 studies and only miR-21/375 was suitable for pooled analysis. Wang et al. [33] systematically analyzed miR21 expression in 16 pairs of ESCC and the corresponding matched nonmalignant adjacent tissues. The author showed that miR-21 downregulation inhibits cell growth and invasion and induces cells to apoptosis by targeting FASL, TIMP3, and RECK genes. Interestingly, suppression or knockdown of miR-21 could induce apoptosis and repress cell proliferation and invasion [34, 35]. One conclusion of the Hamano's [34] study was that miRNAs (including miR-21) that regulate stem cell function are involved in resistance to chemotherapy in esophageal cancer [34]. Ma et al. [35] investigated the role of microRNA-21 (miR-21) and its regulation on phosphatase and tensin homolog deleted from chromosome-10 (PTEN) in esophageal cancer and demonstrated that miR-21 was overexpressed in vitro and ESCC patients, and promoted cell proliferation by targeting PTEN at posttranscriptional level. Furthermore, miR-21 is known as an oncogenic miRNA that is increased in esophageal cancer, and overexpression of miR-21 plays important roles in increasing cell proliferation, migration, invasion, and survival $[33,36]$. The metaanalysis showed that elevated miR-21 (pooled HR $=1.84$ ) expression indeed predict poor OS in patients with ESCC. Although the number of associated studies dealing with miR21 was less than 8 , the available data have shown that miR21 was associated with the prognosis of ESCC, malignant tumor of the digestion system. However, the conclusions should be tempered because the pooled risks of miR-21 for survival, although statistically significant, were not strong, with global HRs of 1.84. The promoter of miR-375 was frequently hypermethylated in EC and miR-375 is a negative regulator of 3-phosphoinositide-dependent protein kinase1 (PDK1) in EC [37]. Besides, the downregulation of miR375 was frequently detected in primary ESCC, which was significantly correlated with advanced stage, distant metastasis. MiR-375 could interact with the $3^{\prime}$-untranslated region of IGF1R and downregulate its expression. Functional assays demonstrated that miR-375 could inhibit clonogenicity, cell motility, cell proliferation, tumor formation, and metastasis in mice [38]. This finding is consistent with Mathe's [39] finding that reduced levels of miR-375 are associated with worse prognosis. Pooled HR of upregulated miR-375 for OS in ESCC patients were 0.55 , indicating that low level of miR375 has a negative impact on OS. The predicted targets of miR-145 were oncogenes myc myelocytomatosis viral-related oncogene (MYCN), FOS, and yamaguchi sarcoma oncogene (YES); cell-cycle promoters such as cyclins D2 and L1; and mitogen-activated protein kinase (MAPK) transduction proteins such as MAP3K3 and MAP4K4 [40]. Kano et al. [41] revealed that miR-145 inhibits cell proliferation and invasion when overexpressed in esophageal carcinoma cell lines in vitro, possibly through downregulation of the FSCN-1 gene product. The transfection of human esophageal carcinoma cells with miR-145 expression plasmids resulted in a greater inhibition of cell mobility; however, the protein level of the previously reported target of miR-145, FSCN1 did not show any significant downregulation [42]. Intriguingly, patients with high levels of miR-145 in the posttreatment biopsy specimens had significantly shorter median disease-free survival (DFS) than did those with low levels [43]. The result is consistent with Feber's [44] finding that high expression of miR-145 is associated with worse survival. Meta-analysis was not performed for miR-145 due to the fact that different EC histology and survival analysis were analysed. The role of miR-145 in EC prognosis remains unclear, although the included studies suggested that miR-145 could be a potential prognostic biomarker for esophageal cancer. Therefore we strongly suggest conducting more prognostic studies for abnormal expression of miR-145 in esophageal carcinomas.

There were several limitations inherent to our analysis. First, although miR-21 has been widely investigated, unfortunately, only seven studies were eligible for pooled analysis. Because of a lack of studies, the limited sample size, and missing data, we could not evaluate subgroups for the sample type, race, and many other factors, especially AD. Furthermore, miR-21 has been found upregulated in patients with non-small cell lung cancer, breast cancer [45], pancreatic cancer [46], and hematological malignancies [47]. Therefore, miR-21 may be a general biomarker for cancer rather than specific for esophageal cancer, although the level of expression may still have prognostic benefits. Second, since the number of studies for miR-375 was less than 7 , it might weaken the reliability of our conclusion. A welldesigned clinical study with large cases of each miRNAs should be performed in the future to validate the relationship 


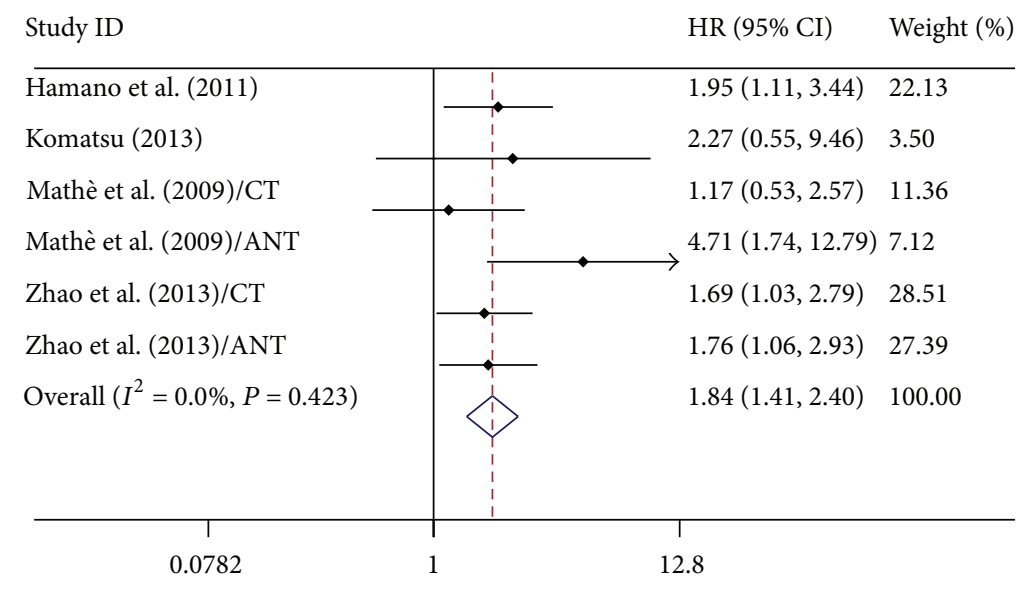

(a)

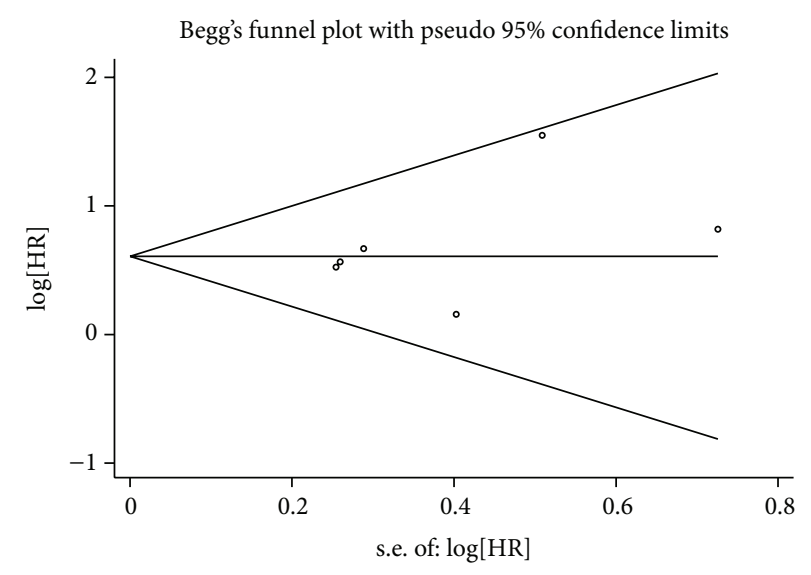

(b)
Meta-analysis estimates, given named study is omitted

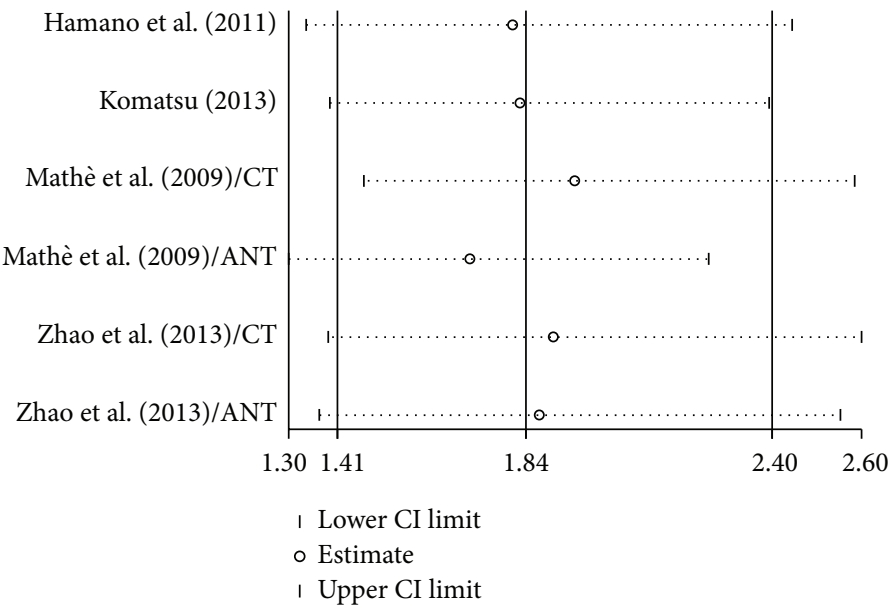

(c)

FIGURE 1: (a) Forest plots of studies evaluating HR of survivals comparing high and low miR-21 expression. (b) Funnel plots of publication bias for meta-analysis of miR-21. (c) Sensitivity analysis for meta-analysis of miR-21.

between miR-21/375/145 expression level and prognosis of EC patients. Third, a clear definition should be made about the cutoff value of miRNAs level for outcomes in ESCC. To date, most investigators use median or mean value in their studies as the cutoff value and the accurate value were different. Fourth, although there was no significant evidence of publication bias in this analysis, cautions should be taken because only studies published in English were selected, which could definitely cause language bias. And the tendency for journals to publish positive results could also make certain bias [28]. Furthermore, tissues are more widely used for miRNA study. However, circulating markers are more acceptable than tissue markers because they can be assayed before treatment and be monitored throughout the life [17]. MiRNAs are considerably resistant to RNase digestion. Also, samples treated under harsh conditions, such as boiling, low/high $\mathrm{pH}$, extended storage, and freezethaw cycles, yielded no significant differences compared to nontreated serum; thereby they are ideal biomarkers for clinical usage [48]. Three studies [31, 32, 49] included in this analysis examined the miRNAs expression levels in in serum/plasma. Thus, the simultaneous detection of miRNAs might serve as a tool to assess the "real-time" status of patients. It is hoped that detection of miRNAs during patient follow-ups would offer the opportunity for early intervention. More studies should be conducted in future to evaluate the prognostic value of miRNA level in serum/plasma. Besides, a group of miRNAs might be better than a single miRNA. Zhao et al. [17] suggest that expression patterns of miR21, miR-181b, and miR-146b, alone or in combination with inflammatory risk score can be used as prognostic classifiers for patients with ESCC [17]. Komatsu et al. [32] revealed that the presence of high miR-21 and low miR-375 concentrations in plasma was an independent prognostic factor. The study of a set of miRNAs associated with prognosis for esophageal cancer is certainly warranted. 


\begin{tabular}{|c|c|c|c|}
\hline \multicolumn{2}{|l|}{ Study ID } & \multirow{2}{*}{$\frac{\operatorname{HR}(95 \% \mathrm{CI})}{0.07(0.00,5916.80)}$} & \multirow{2}{*}{$\frac{\text { Weight (\%) }}{0.09}$} \\
\hline Komatsu et al. (2012) & $\begin{array}{ll} \\
1 \\
1\end{array}$ & & \\
\hline Kong et al. (2012) & 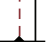 & $0.54(0.20,1.45)$ & 7.30 \\
\hline Li, J (2013) & 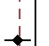 & $0.51(0.31,0.84)$ & 28.83 \\
\hline Mathè et al. (2009)/CT & 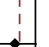 & $0.46(0.20,1.05)$ & 10.42 \\
\hline Mathè et al. (2009)/ANT & 1 & $0.56(0.25,1.25)$ & 11.06 \\
\hline Wu et al. (2014) & 1 & $0.61(0.40,0.91)$ & 42.31 \\
\hline Overall $\left(I^{2}=0.0 \%, P=0.984\right)$ & $\begin{array}{l}1 \\
1 \\
1 \\
1 \\
1 \\
1 \\
1\end{array}$ & $0.55(0.42,0.72)$ & 100.00 \\
\hline 1 & T & 1 & \\
\hline $1.0 e-04$ & 1 & 10000 & \\
\hline
\end{tabular}

(a)

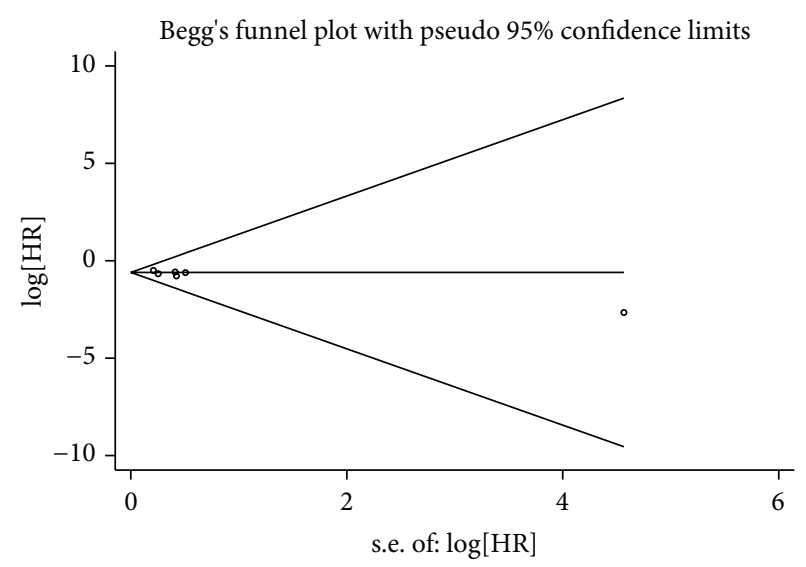

(b)

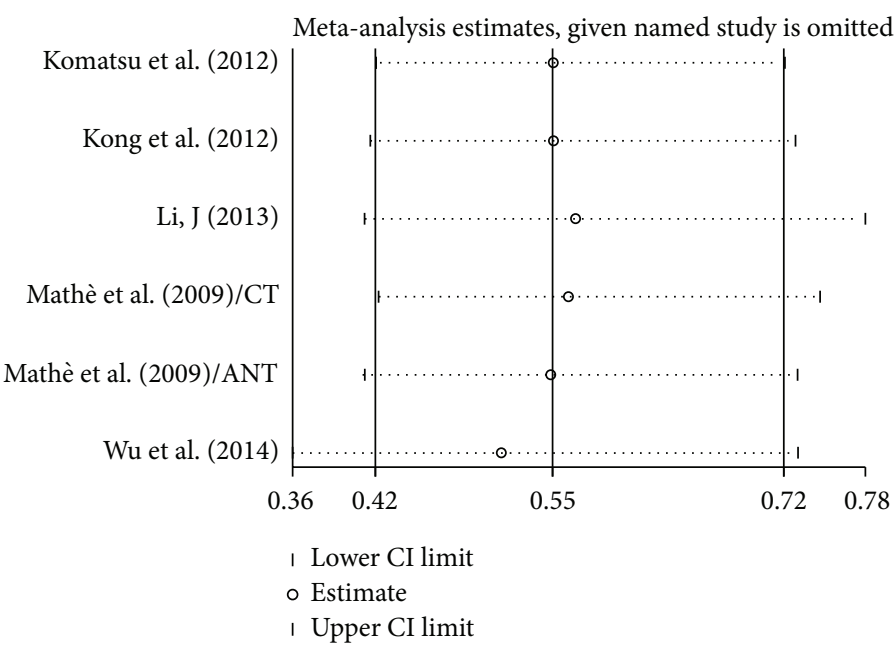

(c)

Figure 2: (a) Forest plots of studies evaluating hazard ratios (HRs) of high miR375 expression as compared to low expression. (b) Funnel plots of publication bias for meta-analysis of miR-375. (c) Sensitivity analysis for meta-analysis of miR-375.

\section{Conclusion}

Despite the limitations described above, our results support that miR-21 and miR-375 have a prognostic role in ESCC and therefore may be useful therapeutic targets for the treatment of ESCC and meticulous follow-up for early detection of recurrence. Validation of the results of these miRNAs by independent and prospective study is certainly warranted, and it is hoped that this analysis highlights the most appropriate candidates for their clinical significance and role in the prognosis of esophageal cancer.

\section{Abbreviations}

EC:

AD: $\quad$ Esophageal adenocarcinoma

ESCC: Esophageal squamous cell carcinoma

miRNAs: MicroRNAs

OS: Overall survival

CI: Confidence interval

HR: Hazard ratios.

\section{Conflict of Interests}

The authors declare that they have no conflict of interests regarding the publication of this paper.

\section{Acknowledgments}

The authors would like to acknowledge the help from Second/First Hospital Affiliated to Medical College of Shihezi University. The work was supported in part by doctor/scientific Grant 2014BB019 from Xin jiang Production and Construction Corps and NFSC 81260301 and 81160301 from the National Science Foundation of China.

\section{References}

[1] J. Lagergren, R. Bergström, A. Lindgren, and O. Nyrén, “Symptomatic gastroesophageal reflux as a risk factor for esophageal adenocarcinoma," The New England Journal of Medicine, vol. 340, no. 11, pp. 825-831, 1999. 
[2] P. C. Enzinger and R. J. Mayer, "Esophageal Cancer," The New England Journal of Medicine, vol. 349, no. 23, pp. 2241-2252, 2003.

[3] A. Jemal, F. Bray, M. M. Center, J. Ferlay, E. Ward, and D. Forman, "Global cancer statistics," CA Cancer Journal for Clinicians, vol. 61, no. 2, pp. 69-90, 2011.

[4] M. Sant, T. Aareleid, F. Berrino et al., "EUROCARE-3: Survival of cancer patients diagnosed 1990-94-results and commentary," Annals of Oncology, vol. 14, supplement 5, pp. v61-v118, 2003.

[5] J. McCann, "Esophageal cancers: changing character, increasing incidence.," Journal of the National Cancer Institute, vol. 91, no. 6, pp. 497-498, 1999.

[6] M. B. Orringer, "Multimodality therapy for esophageal carcinoma-update," Chest, vol. 103, no. 4, pp. 406S-409S, 1993.

[7] D. P. Bartel, "MicroRNAs: genomics, biogenesis, mechanism, and function," Cell, vol. 116, no. 2, pp. 281-297, 2004.

[8] J. S. Mattick and I. V. Makunin, "Small regulatory RNAs in mammals," Human Molecular Genetics, vol. 14, no. 1, pp. R121R132, 2005.

[9] V. N. Kim, "MicroRNA biogenesis: coordinated cropping and dicing," Nature Reviews Molecular Cell Biology, vol. 6, no. 5, pp. 376-385, 2005.

[10] G. Hutvágner and P. D. Zamore, "A microRNA in a multipleturnover RNAi enzyme complex," Science, vol. 297, no. 5589, pp. 2056-2060, 2002.

[11] J. Winter, S. Jung, S. Keller, R. I. Gregory, and S. Diederichs, "Many roads to maturity: microRNA biogenesis pathways and their regulation," Nature Cell Biology, vol. 11, no. 3, pp. 228-234, 2009.

[12] G. A. Calin, C. Sevignani, C. D. Dumitru et al., "Human microRNA genes are frequently located at fragile sites and genomic regions involved in cancers," Proceedings of the National Academy of Sciences of the United States of America, vol. 101, no. 9, pp. 2999-3004, 2004.

[13] A. J. Schetter, S. Y. Leung, J. J. Sohn et al., "MicroRNA expression profiles associated with prognosis and therapeutic outcome in colon adenocarcinoma," Journal of the American Medical Association, vol. 299, no. 4, pp. 425-436, 2008.

[14] N. Yanaihara, N. Caplen, E. Bowman et al., "Unique microRNA molecular profiles in lung cancer diagnosis and prognosis," Cancer Cell, vol. 9, no. 3, pp. 189-198, 2006.

[15] M. V. Iorio, M. Ferracin, C.-G. Liu et al., "MicroRNA gene expression deregulation in human breast cancer," Cancer Research, vol. 65, no. 16, pp. 7065-7070, 2005.

[16] M. V. Iorio, R. Visone, G. Di Leva et al., "MicroRNA signatures in human ovarian cancer," Cancer Research, vol. 67, no. 18, pp. 8699-8707, 2007.

[17] Y. Zhao, A. J. Schetter, G. B. Yang et al., "MicroRNA and inflammatory gene expression as prognostic marker for overall survival in esophageal squamous cell carcinoma," International Journal of Cancer, vol. 132, no. 12, pp. 2901-2909, 2013.

[18] T. Zhang, Q. Wang, D. Zhao et al., "The oncogenetic role of microRNA-31 as a potential biomarker in oesophageal squamous cell carcinoma," Clinical Science, vol. 121, no. 10, pp. 437447, 2011.

[19] D. F. Stroup, J. A. Berlin, S. C. Morton et al., "Meta-analysis of observational studies in epidemiology: a proposal for reporting," Journal of the American Medical Association, vol. 283, no. 15, pp. 2008-2012, 2000.
[20] D. Moher, A. Liberati, J. Tetzlaff, and D. G. Altman, "Preferred reporting items for systematic reviews and meta-analyses: the PRISMA statement," British Medical Journal, vol. 339, article b2535, 2009.

[21] L. Zhang, S. Riethdorf, G. Wu et al., "Meta-analysis of the prognostic value of circulating tumor cells in breast cancer," Clinical Cancer Research, vol. 18, no. 20, pp. 5701-5710, 2012.

[22] J. F. Tierney, L. A. Stewart, D. Ghersi, S. Burdett, and M. R. Sydes, "Practical methods for incorporating summary time-toevent data into meta-analysis," Trials, vol. 8, article 16, 2007.

[23] R. DerSimonian and N. Laird, "Meta-analysis in clinical trials," Controlled Clinical Trials, vol. 7, no. 3, pp. 177-188, 1986.

[24] J. P. T. Higgins, S. G. Thompson, J. J. Deeks, and D. G. Altman, "Measuring inconsistency in meta-analyses," British Medical Journal, vol. 327, no. 7414, pp. 557-560, 2003.

[25] B. E. G. Rothberg, M. B. Bracken, and D. L. Rimm, “Tissue biomarkers for prognosis in cutaneous melanoma: a systematic review and meta-analysis," Journal of the National Cancer Institute, vol. 101, no. 7, pp. 452-474, 2009.

[26] B. G. Koerkamp, N. N. Rahbari, M. W. Büchler, M. Koch, and J. Weitz, "Circulating tumor cells and prognosis of patients with resectable colorectal liver metastases or widespread metastatic colorectal cancer: a meta-analysis," Annals of Surgical Oncology, vol. 20, no. 7, pp. 2156-2165, 2013.

[27] M. Egger, G. D. Smith, M. Schneider, and C. Minder, "Bias in meta-analysis detected by a simple, graphical test," British Medical Journal, vol. 315, pp. 629-634, 1997.

[28] S. Lin, L. Pan, S. Guo et al., "Prognostic role of microRNA181a/b in hematological malignancies: a meta-analysis," PLoS ONE, vol. 8, no. 3, Article ID e59532, 2013.

[29] R. Hummel, D. J. Hussey, M. Z. Michael et al., "MiRNAs and their association with locoregional staging and survival following surgery for esophageal carcinoma," Annals of Surgical Oncology, vol. 18, no. 1, pp. 253-260, 2011.

[30] L. Hong, Y. Han, H. Zhang et al., "The prognostic and chemotherapeutic value of miR-296 in esophageal squamous cell carcinoma," Annals of Surgery, vol. 251, no. 6, pp. 1056-1063, 2010.

[31] K. Tanaka, H. Miyata, M. Yamasaki et al., "Circulating miR200c levels significantly predict response to chemotherapy and prognosis of patients undergoing neoadjuvant chemotherapy for esophageal cancer," Annals of Surgical Oncology, vol. 20, supplement 3, pp. 607-615, 2013.

[32] S. Komatsu, D. Ichikawa, H. Takeshita et al., "Prognostic impact of circulating miR-21 and miR-375 in plasma of patients with esophageal squamous cell carcinoma," Expert Opinion on Biological Therapy, vol. 12, supplement 1, pp. S53-S59, 2012.

[33] N. Wang, C. Zhang, J. He et al., "MiR-21 down-regulation suppresses cell growth, invasion and induces cell apoptosis by targeting FASL, TIMP3, and RECK genes in esophageal carcinoma," Digestive Diseases and Sciences, vol. 58, no. 7, pp. 1863-1870, 2013.

[34] R. Hamano, H. Miyata, M. Yamasaki et al., "Overexpression of miR-200c induces chemoresistance in esophageal cancers mediated through activation of the Akt signaling pathway," Clinical Cancer Research, vol. 17, no. 9, pp. 3029-3038, 2011.

[35] W.-J. Ma, G.-D. Lv, A. Tuersun et al., "Role of microRNA21 and effect on PTEN in Kazakh's esophageal squamous cell carcinoma," Molecular Biology Reports, vol. 38, no. 5, pp. 32533260,2011 . 
[36] P. Li, W. M. Mao, Z. G. Zheng, Z. M. Dong, and Z.Q. Ling, "Down-regulation of pten expression modulated by dysregulated mir-21 contributes to the progression of esophageal cancer," Digestive Diseases and Sciences, vol. 58, pp. 3483-3493, 2013.

[37] X. Li, R. Lin, and J. Li, "Epigenetic silencing of MicroRNA375 regulates PDK1 expression in esophageal cancer," Digestive Diseases and Sciences, vol. 56, no. 10, pp. 2849-2856, 2011.

[38] K. L. Kong, D. L. W. Kwong, T. H. Chan et al., "MicroRNA-375 inhibits tumour growth and metastasis in oesophageal squamous cell carcinoma through repressing insulin-like growth factor 1 receptor," Gut, vol. 61, no. 1, pp. 33-42, 2012.

[39] E. A. Mathé, H. N. Giang, E. D. Bowman et al., "MicroRNA expression in squamous cell carcinoma and adenocarcinoma of the esophagus: associations with survival," Clinical Cancer Research, vol. 15, no. 19, pp. 6192-6200, 2009.

[40] R. Ogawa, H. Ishiguro, Y. Kuwabara et al., "Expression profiling of micro-RNAs in human esophageal squamous cell carcinoma using RT-PCR," Medical Molecular Morphology, vol. 42, no. 2, pp. 102-109, 2009.

[41] M. Kano, N. Seki, N. Kikkawa et al., "MiR-145, miR-133a and miR-133b: tumor-suppressive miRNAs target FSCN1 in esophageal squamous cell carcinoma," International Journal of Cancer, vol. 127, no. 12, pp. 2804-2814, 2010.

[42] B. L. Wu, L. Y. Xu, Z. P. Du et al., "MiRNA profile in esophageal squamous cell carcinoma: downregulation of miR-143 and miR145," World Journal of Gastroenterology, vol. 17, no. 1, pp. 79-88, 2011.

[43] M. A. Ko, G. Zehong, C. Virtanen et al., "MicroRNA expression profiling of esophageal cancer before and after induction chemoradiotherapy," Annals of Thoracic Surgery, vol. 94, no. 4, pp. 1094-1103, 2012.

[44] A. Feber, L. Xi, A. Pennathur et al., "MicroRNA prognostic signature for nodal metastases and survival in esophageal adenocarcinoma," Annals of Thoracic Surgery, vol. 91, no. 5, pp. 1523-1530, 2011.

[45] L. X. Yan, X. F. Huang, Q. Shao et al., "MicroRNA miR21 overexpression in human breast cancer is associated with advanced clinical stage, lymph node metastasis and patient poor prognosis," RNA, vol. 14, no. 11, pp. 2348-2360, 2008.

[46] M. Dillhoff, J. Liu, W. Frankel, C. Croce, and M. Bloomston, "MicroRNA-21 is overexpressed in pancreatic cancer and a potential predictor of survival," Journal of Gastrointestinal Surgery, vol. 12, no. 12, pp. 2171-2176, 2008.

[47] S. Rossi, M. Shimizu, E. Barbarotto et al., "MicroRNA fingerprinting of CLL patients with chromosome 17p deletion identify a $m i R-21$ score that stratifies early survival," Blood, vol. 116, no. 6, pp. 945-952, 2010.

[48] X. Chen, Y. Ba, L. Ma et al., "Characterization of microRNAs in serum: a novel class of biomarkers for diagnosis of cancer and other diseases," Cell Research, vol. 18, no. 10, pp. 997-1006, 2008.

[49] C. Wu, M. Li, C. Hu, and H. Duan, "Clinical significance of serum mir-223, mir-25 and mir-375 in patients with esophageal squamous cell carcinoma," Molecular Biology Reports, vol. 41, no. 3, pp. 1257-1266, 2014. 


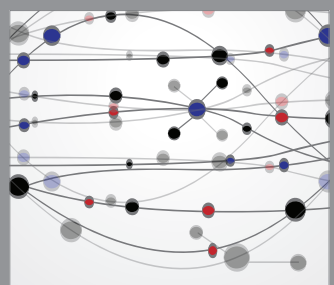

The Scientific World Journal
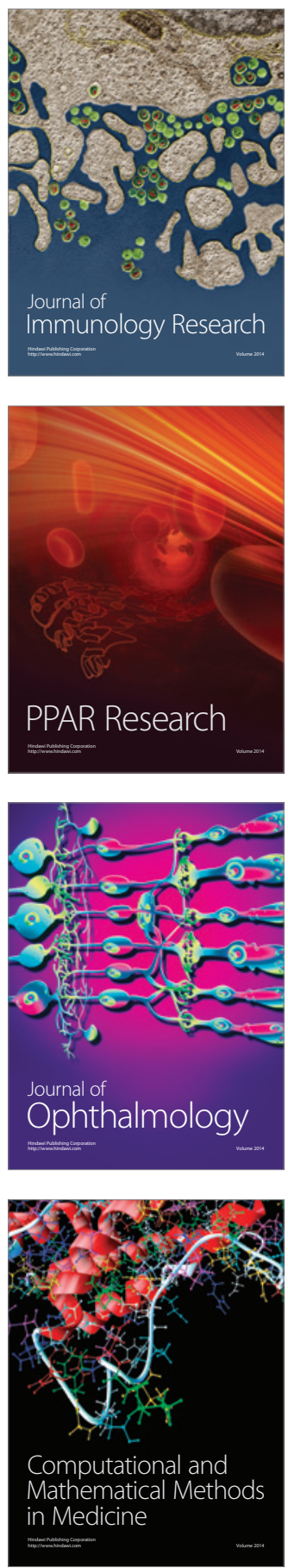

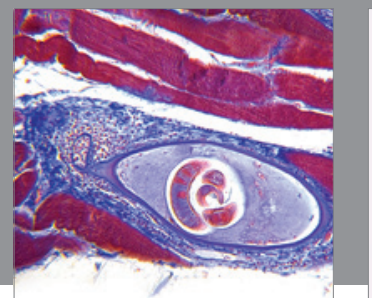

Gastroenterology

Research and Practice
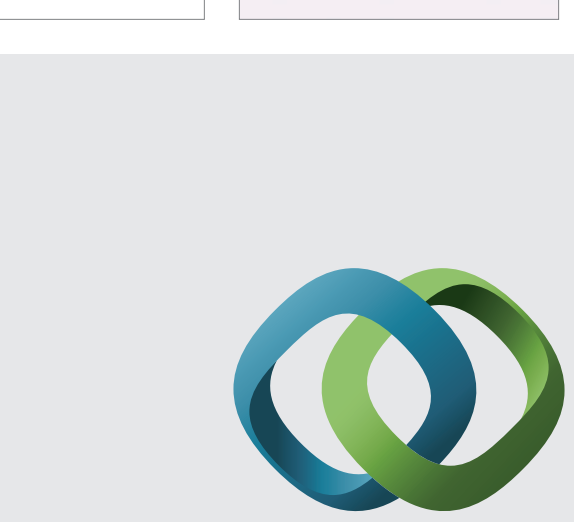

\section{Hindawi}

Submit your manuscripts at

http://www.hindawi.com
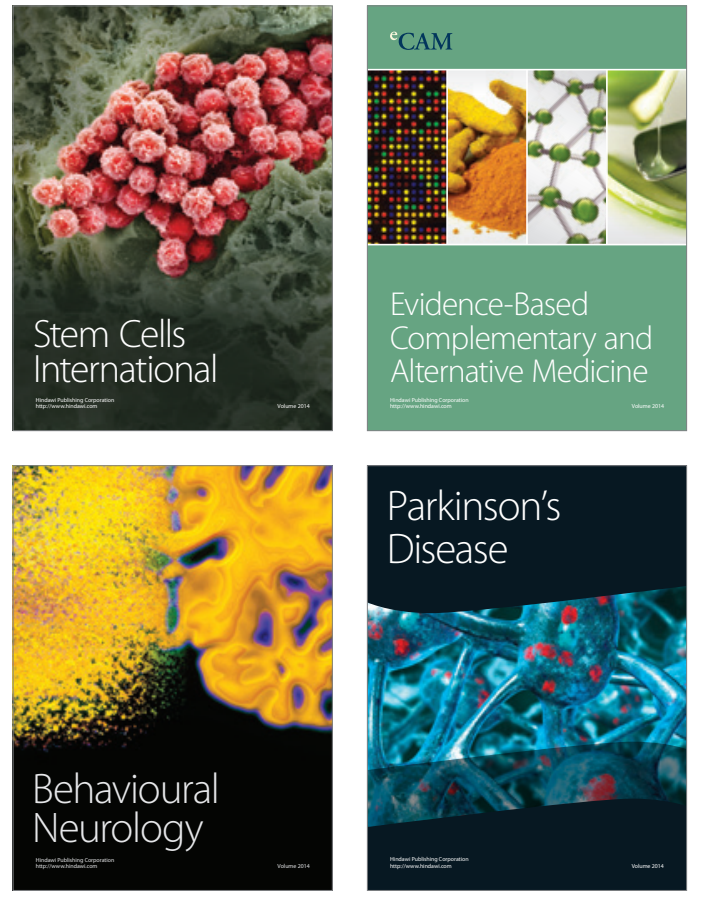
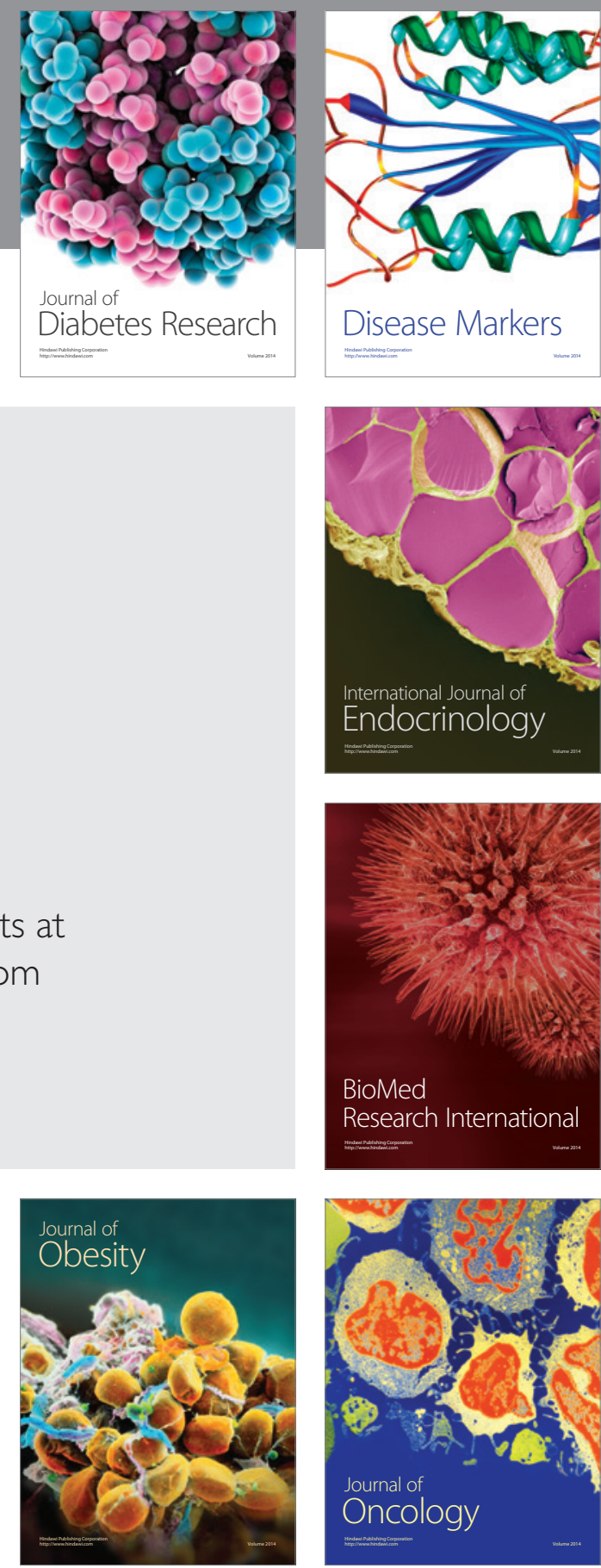

Disease Markers
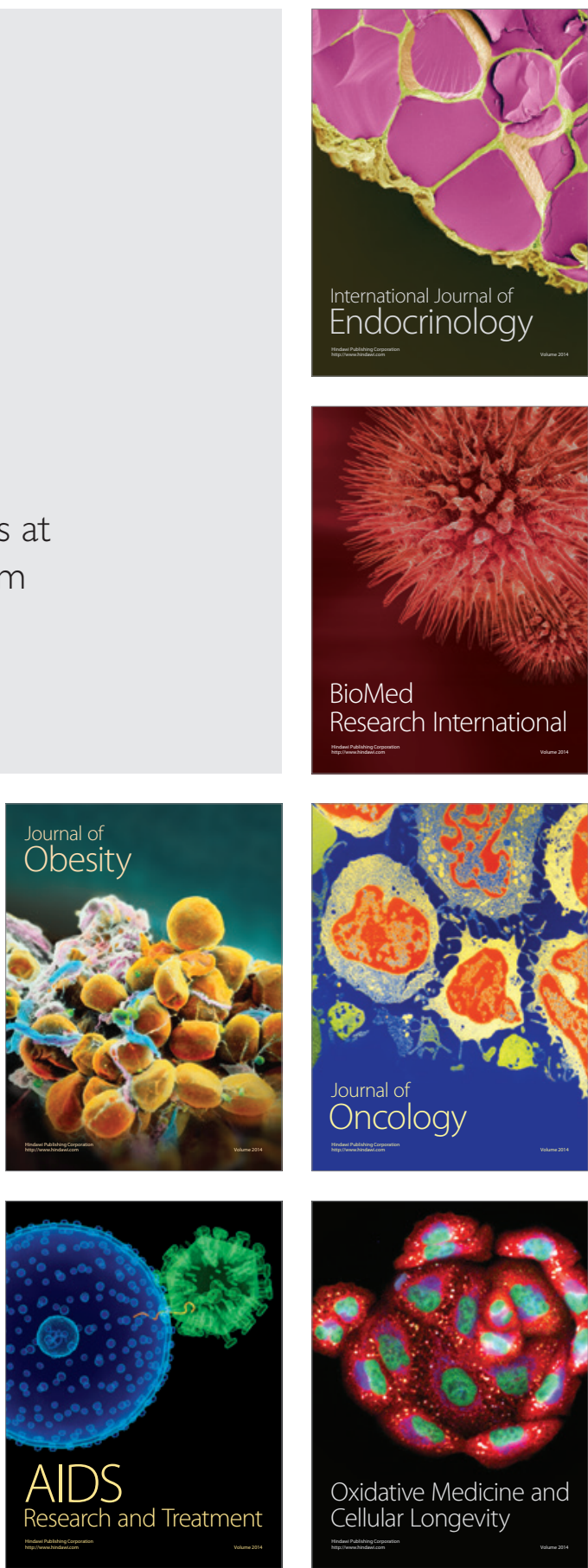